\title{
Development of a Scalable Synthesis of a Common
}

\section{Eastern Tricyclic Lactone for Construction of the}

\section{Nodulisporic Acids}

Amos B. Smith, III*, László Kürti, Akin H. Davulcu and Young Shin Cho

Department of Chemistry, Monell Chemical Senses Center and Laboratory for Research on the Structure of Matter, University of Pennsylvania, Philadelphia, PA 19104

\author{
smithab@sas.upenn.edu
}

\section{SUPPORTING INFORMATION}




\section{MATERIALS AND METHODS}

Except as otherwise indicated, all reactions were carried out under an argon atmosphere in flame-dried glassware, and solvents were freshly distilled. The argon was deoxygenated and dried by passage through an OXICLEAR ${ }^{\mathrm{TM}}$ filter from Aldrich and Drierite tube, respectively. Diethyl ether $\left(\mathrm{Et}_{2} \mathrm{O}\right)$ and tetrahydrofuran (THF) were distilled from sodium / benzophenone. Dichloromethane $\left(\mathrm{CH}_{2} \mathrm{Cl}_{2}\right)$ was distilled from calcium hydride. $n$-Butyllithium, $s$-butyllithium, and $t$-butyllithium were purchased from Aldrich and titrated against $N$-benzylbenzamide ${ }^{1}$ prior to use.

Except as indicated otherwise, all other reagents were purchased from Aldrich, Acros, or Strem chemicals and used as received. Reactions were monitored by thin-layer chromatography (TLC) with $0.25 \mathrm{~mm}$ E. Merck pre-coated silica gel plates. Silica gel for flash chromatography (particle size 0.040$0.063 \mathrm{~mm}$ ) was supplied by Silicycle and Sorbent Technologies. Yields refer to chromatographically and spectroscopically pure compounds, unless otherwise stated. All melting points were determined on a Bristoline heated-stage microscope or Thomas Hoover apparatus and are uncorrected. Infrared spectra were recorded on either a Perkin-Elmer Model 283B, Perkin-Elmer Model 1600 FTIR, or Jasco FTIR480plus spectrometer. ${ }^{1} \mathrm{H}-\mathrm{NMR}$ and ${ }^{13} \mathrm{C}-\mathrm{NMR}$ spectra were recorded on a Bruker AMX-500 spectrometer. Chemical shifts are reported as $\delta$ values relative to internal $\mathrm{CDCl}_{3}\left(\delta 7.26\right.$ for ${ }^{1} \mathrm{H}, \delta 77.0$ for $\left.{ }^{13} \mathrm{C}\right), \mathrm{C}_{6} \mathrm{D}_{6}\left(\delta 7.15\right.$ for ${ }^{1} \mathrm{H}, \delta 128.4$ for $\left.{ }^{13} \mathrm{C}\right)$ or $\mathrm{CD}_{3} \mathrm{OD}\left(\delta 4.87,3.31\right.$ for ${ }^{1} \mathrm{H}, \delta 49.15$ for $\left.{ }^{13} \mathrm{C}\right)$. Optical rotations were obtained with a Perkin-Elmer model 241 polarimeter in the solvent indicated. High resolution mass spectra were measured on either a VG Micromass 70/70H or VG ZAB-E spectrometer. 


\section{DETAILED EXPERIMENTAL PROCEDURES}

\section{Preparation of (S)-8a'-methyl-3',4',8',8a'-tetrahydro-2'H-spiro[[1,3]dioxolane-2,1'-naphthalen] 6'(7'H)-one [(+)-10].}

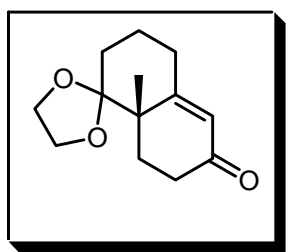

Compound (+)-10 was prepared according to the procedure described by J. Demnitz et al. $^{2}$ We have conducted this reaction on a $50 \mathrm{~g}$ scale without seeing diminished yields compared to the literature precedent above.

Large-scale procedure: A 3 L round bottom flask was charged with $(+)$-Wieland-Miescher ketone $\left(50.00 \mathrm{~g}, 281 \mathrm{mmol}, 1\right.$ equiv; $[\alpha]_{\mathrm{D}}^{20}+110.0^{\circ}$ at $\left.c 1.11, \mathrm{CHCl}_{3}\right)$ and ethylene glycol $(1405 \mathrm{~mL} ; \mathrm{d}=1.13$; 90 equiv). Next, in one portion $p-\mathrm{TsOH} \cdot \mathrm{H}_{2} \mathrm{O}(53.5 \mathrm{~g}, 281 \mathrm{mmol} ; 1$ equiv) was also added and the resulting mixture was stirred for exactly 23 minutes at room temperature. The reaction mixture was then poured into a mixture of ice and saturated aqueous $\mathrm{NaHCO}_{3}$ solution $(3000 \mathrm{~mL})$. The resulting solution was extracted with EtOAc $(4 \times 500 \mathrm{~mL})$. The combined organic layers were washed with brine, dried over $\mathrm{MgSO}_{4}$ and concentrated in vacuo. The crude product was recrystallized using Hexanes : $\mathrm{Et}_{2} \mathrm{O}=$ 20:1. The product was obtained as white crystals: $56.08 \mathrm{~g}(90 \%) . \mathrm{mp}=52{ }^{\circ} \mathrm{C}\left(\right.$ lit. $\left.^{2} 51-52{ }^{\circ} \mathrm{C}\right) . \underline{\text { Remark: }}$ The purification has to be done with great care to make sure that the bis-ketal side-product is completely separated from the desired product as verified by NMR. Any remaining bis-ketal will be difficult to separate from the product of the next reaction $[(+)-8]$.

The ${ }^{1} \mathrm{H}-\mathrm{NMR}$ data was in complete agreement with the reported values. ${ }^{3}$ 
Preparation of (S)-8a'-methyl-5'-(phenylthiomethyl)-3',4',8',8a'-tetrahydro-2'H-spiro[[1,3] dioxolane-2,1'-naphthalen]-6'(7'H)-one [(+)-8]

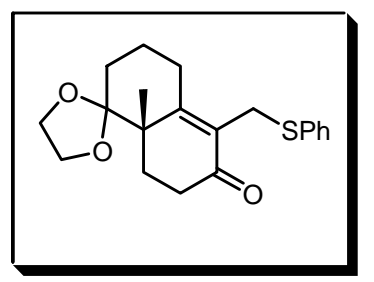

A $1 \mathrm{~L}$ round bottom flask, equipped with a reflux condenser, was charged with $(+)-8(81.76 \mathrm{~g}, 368.1 \mathrm{mmol} ; \mathrm{FW}=222.13)$, thiophenol $(60.78 \mathrm{~g}, 56.40 \mathrm{~mL}, 1.5$ equiv), $37 \%(\mathrm{wt} / \mathrm{wt})$ aqueous formaldehyde solution $(48.36 \mathrm{~g}, 44.36 \mathrm{~mL}, 1.63$ equiv), triethylamine ( $46.82 \mathrm{~g}, 64.48 \mathrm{~mL}, 1.26$ equiv) and absolute ethanol $(281 \mathrm{~mL})$. The resulting mixture was stirred at room temperature under Ar atmosphere until a homogeneous solution resulted. The reaction mixture was then heated at reflux for 4 days. The progress of the reaction was monitored by TLC (Hexanes:EtOAc $=2: 1$ where the product has an $\mathrm{R}_{\mathrm{f}}$ value of 0.6 ). The reaction mixture was cooled to room temperature. The large amount of needle-like crystals were filtered and set aside. The mother liquor was then treated with $5 \%$ aqueous $\mathrm{KOH}$ solution $(1000 \mathrm{~mL})$ and extracted with $\mathrm{Et}_{2} \mathrm{O}(3 \mathrm{x}$ $400 \mathrm{~mL})$. The combined organic layers were washed with $5 \%$ aqueous $\mathrm{KOH}$ solution $(3 \times 200 \mathrm{~mL})$, dried over $\mathrm{MgSO}_{4}$ and concentrated in vacuo. The filtered crystals and the crude product were taken up in a minimum quantity of $\mathrm{Et}_{2} \mathrm{O}$ then hexanes were added to obtain a cloudy solution. The solution then was allowed to cool to room temperature where in a couple of hours a large quantity of crystals precipitated. The off-white crystals were filtered and air-dried: $113.5 \mathrm{~g}(89 \%)$.

The ${ }^{1} \mathrm{H}-\mathrm{NMR}$ data was in complete agreement with the reported values. ${ }^{4}$ 


\section{Preparation of $\left(4 a^{\prime} R, 5\right.$ 'R,8a'S)-5'-(hydroxymethyl)-5',8a'-dimethylhexahydro-2'H-spiro[[1,3]}

dioxolane-2,1'-naphthalen]-6'(7'H)-one [(-)-13].

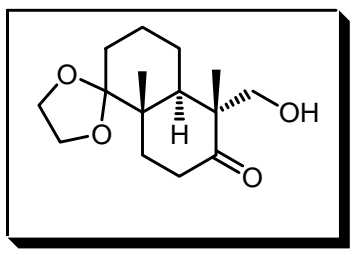

Birch reduction: A $1 \mathrm{~L}$ three-necked flask, equipped with a mechanical stirrer and a cold-finger condenser outfitted with a gas adapter, was charged with liquid ammonia $(500 \mathrm{~mL})$. The liquid ammonia was condensed slowly by cooling the bottom of the flask to $-78^{\circ} \mathrm{C}$ with acetone/dry ice and slowly passing ammonia gas through the coldfinger that was also at $-78^{\circ} \mathrm{C}$. The rate of the ammonia flow is monitored by a gas bubbler. (Warning: As the ammonia gas condenses, the pressure will drop in the apparatus, potentially leading to backsuction, therefore one must adjust the main valve of the ammonia tank to maintain an even flow of the ammonia gas) Once approximately $500 \mathrm{~mL}$ of liquid ammonia was collected, the ammonia line was replaced with an Ar line and a steady stream of Ar was maintained to avoid moisture seeping into the apparatus. Next, lithium metal wire (2.42 g, $348 \mathrm{mmol}, 6$ equiv) was cut to $1 \mathrm{~cm}$ pieces and washed twice with hexanes $(2 \times 100 \mathrm{~mL})$. The lithium wire pieces were added individually to the vigorously stirred liquid ammonia. Almost instantaneously the colorless liquid ammonia turned dark blue. (Note: It takes approximately one hour to completely dissolve $2.5 \mathrm{~g}$ of Li metal.) Once all of the lithium metal was dissolved, the solution of $\alpha, \beta$-unsaturated ketone $(+)-8(20.00 \mathrm{~g}, 58.06 \mathrm{mmol}, 1$ equiv) and $t-\mathrm{BuOH}$ (10.8 $\mathrm{mL}, 116 \mathrm{mmol}, 2$ equiv) in THF $(200 \mathrm{~mL})$ was added to the $\mathrm{Li} / \mathrm{NH}_{3}(1)$ via a cannula over 40 minutes. The reaction mixture was then allowed to stir vigorously for one hour at $-78{ }^{\circ} \mathrm{C}$. Next, the unreacted $\mathrm{Li} / \mathrm{NH}_{3}(\mathrm{l})$ was destroyed by slowly(!) adding isoprene $(13.6 \mathrm{~mL}, 136 \mathrm{mmol}, 2.4$ equiv) to the reaction mixture. (Warning: If the isoprene is not added in a carefully controlled manner, a runaway reaction may result which is capable of destroying of the entire apparatus. This is due to the extreme exothermic nature of the reaction of isoprene with lithium metal. If heat is not dissipated efficiently, the liquid ammonia reaches its boiling point $\left(-33^{\circ} \mathrm{C}\right)$ quickly, resulting in a rapid increase of pressure.) 
Upon destroying the excess lithium, the originally dark blue solution turned into a colorless solution.

Next, the cooling bath and the cold-finger condenser were removed and a 24/40 gas adapter was fitted into the three-necked flask and a constant but rapid stream of Ar was blown through the apparatus to evaporate all of the liquid ammonia (2-3 h). (The outgoing Ar stream was continuously checked for ammonia content using a glass rod previously immersed into concentrated $\mathrm{HCl}$ solution; a massive white cloud of $\mathrm{NH}_{4} \mathrm{Cl}$ was formed if the test was positive.) Upon the complete removal of the ammonia, the residual THF solution of the lithium enolate (that was somewhat cloudy) was dissolved in freshly distilled THF $(150 \mathrm{~mL})$. Next, the reaction mixture was cooled to $0{ }^{\circ} \mathrm{C}$. In the meantime freshly distilled TMSCl (40.84 mL, $322 \mathrm{mmol}, 5.54$ equiv) and $\mathrm{Et}_{3} \mathrm{~N}$ (44.80 mL, $322 \mathrm{mmol}, 5.54$ equiv) were dissolved in THF $(200 \mathrm{~mL})$ and stirred for 20 minutes. Once the white precipitate settled on the bottom of the flask, the clear $\mathrm{TMSCl} / \mathrm{Et}_{3} \mathrm{~N} / \mathrm{THF}$ solution was cannulated over to the reaction mixture containing the lithium enolate in $\mathrm{THF}$ at $0{ }^{\circ} \mathrm{C}$. The progress of the reaction was monitored by TLC (Hexanes : EtOAc $\left.=5: 1, \mathrm{R}_{\mathrm{f}}=0.8\right)$. The reaction mixture was then poured into a mixture of $\mathrm{Et}_{2} \mathrm{O}(500 \mathrm{~mL})$ and saturated aqueous solution of $\mathrm{NaHCO}_{3}(500 \mathrm{~mL})$. The combined organic layers were washed with aqueous $\mathrm{NaHCO}_{3}(3 \times 200 \mathrm{~mL})$, brine $(3 \times 100 \mathrm{~mL})$, dried over $\mathrm{MgSO}_{4}$ and concentrated in vacuo. The crude TMS enol ether 12 was passed through a pad of basic alumina (200 g) to give a pale yellow oil: $15.3 \mathrm{~g}$ (85\% yield).

Sc-Triflate Catalyzed Hydroxymethylation: A 1 L round bottom flask was charged with TMS enol ether 12 (15.3 g, $49.2 \mathrm{mmol}, 1$ equiv), THF (350 mL) and 37\% aqueous formaldehyde solution (150 mL). To the resulting solution, $\mathrm{Sc}(\mathrm{OTf})_{3}(2.18 \mathrm{~g}, 4.43 \mathrm{mmol}, 0.09$ equiv) was added in one portion and the resulting solution was stirred for $1 \mathrm{~h}$ at room temperature. The progress of the reaction was monitored by TLC $\left(\mathrm{CHCl}_{3}: \mathrm{MeOH}=125: 1, \mathrm{R}_{\mathrm{f}}=0.35\right)$. Next, EtOAc $(250 \mathrm{~mL})$ was added to the reaction mixture. The resulting aqueous phase was extracted with EtOAc $(2 \times 150 \mathrm{~mL})$ and the combined organic extracts were washed brine $(3 \times 75 \mathrm{~mL})$, dried over $\mathrm{MgSO}_{4}$ and concentrated in vacuo. The crude product was 
purified using flash chromatography $\left(\mathrm{CHCl}_{3}: \mathrm{MeOH}=125: 1\right)$ and $(-)-\mathbf{1 3}$ was isolated as a thick colorless oil: $10.7 \mathrm{~g}(81 \%) .[\alpha]_{\mathrm{D}}^{20}-44.1^{\mathrm{o}}\left(\mathrm{c} 0.98, \mathrm{CHCl}_{3}\right)$.

IR (neat) 3473.7 (s, br), 2946.5 (s), 2876.1 (s), 1699.5 (s), 1464.0 (w), 1383.0 (w), 1282.7 (w), 1184.1 (m), $1137.1(\mathrm{~m}), 1059.2(\mathrm{~m}), 951.5(\mathrm{w}), 909.8(\mathrm{w}) \mathrm{cm}^{-1} ;{ }^{1} \mathrm{H}$ NMR (500 MHz, $\left.\mathrm{CDCl}_{3}\right) \delta 1.02(\mathrm{~s}, 3 \mathrm{H})$, 1.28, (s, 3 H). 1.40-1.57 (m, 4 H), 1.64-1.75 (m, 3 H), $1.91(\mathrm{dt}, J=4.7,13.9 \mathrm{~Hz}, 1 \mathrm{H}), 2.19$ (dd, $J=3.8$, 11.2 Hz, $1 \mathrm{H}), 2.26(\mathrm{ddd}, J=2.9,4.6,15.2 \mathrm{~Hz}, 1 \mathrm{H}), 2.51(\mathrm{t}, J=7.1 \mathrm{~Hz}, 1 \mathrm{H}), 2.64(\mathrm{dt}, J=6.2,14.9 \mathrm{~Hz}$, $1 \mathrm{H}), 3.40(\mathrm{dd}, J=7.0,11.5 \mathrm{~Hz}, 1 \mathrm{H}), 3.59(\mathrm{dd}, J=7.2,11.5 \mathrm{~Hz}, 1 \mathrm{H}), 3.81-3.95(\mathrm{~m}, 4 \mathrm{H}) ;{ }^{13} \mathrm{C}$ NMR $\left(125 \mathrm{MHz} \mathrm{CDCl}_{3}\right) \delta 218.7,112.7,66.7,65.3,64.9,52.3,43.3,42.5,35.2,30.3,29.9,22.7,21.3,16.8$, 16.3; HRMS (ESI) $m / z 269.1758\left[(\mathrm{M}+\mathrm{H})^{+}\right.$; calcd for $\left.\mathrm{C}_{15} \mathrm{H}_{25} \mathrm{O}_{4}: 269.1753\right]$.

Preparation of (4aS,5R,6S,8aS)-6-(tert-butyldimethylsilyloxy)-5-((tert-butyl-dimethylsilyloxy) methyl)-5,8a-dimethyloctahydronaphthalen-1(2H)-one [(-)-16]

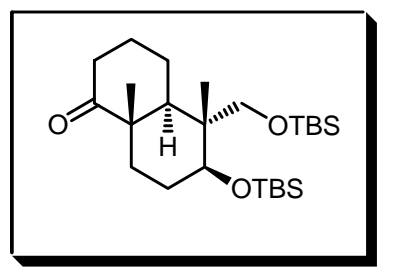

Diastereoselective Hydride Reduction: A 2 L round bottom flask was charged with $\mathrm{Me}_{4} \mathrm{NBH}(\mathrm{OAc})_{3}\left(30.62 \mathrm{~g}, 116 \mathrm{mmol}, 5.12\right.$ equiv), $\mathrm{HPLC}$ grade $\mathrm{CH}_{3} \mathrm{CN}$ $(600 \mathrm{~mL})$ and glacial acetic acid $(121 \mathrm{~mL})$. The resulting suspension was cooled to $-40{ }^{\circ} \mathrm{C}$ with an acetonitrile-dry ice bath then the solution of $(-)-13(6.10 \mathrm{~g}, 22.73 \mathrm{mmol}, 1$ equiv) in acetonitrile $(50 \mathrm{~mL})$ was added over $1 \mathrm{~h}$ while the reaction mixture was vigorously stirred. The reaction mixture was allowed to stir at $-40{ }^{\circ} \mathrm{C}$ for an additional 4 hours then was allowed to warm to room temperature and quenched with saturated Rochelle's salt $(250 \mathrm{~mL})$. EtOAc $(4 \times 150 \mathrm{~mL})$ was used to extract the aqueous phase. The combined organic layers were washed with brine $(2 \times 150 \mathrm{~mL})$, 
dried over $\mathrm{MgSO}_{4}$ and concentrated in vacuo. The crude product (-)-14 was taken to the next step without purification.

Removal of Dioxolane: A $500 \mathrm{~mL}$ round bottom flask was charged with crude diol (-)-14 and THF (150 mL). To this solution, freshly deoxygenated aqueous $2 \mathrm{~N} \mathrm{HCl}(34 \mathrm{~mL})$ was added in one portion and the reaction mixture was vigorously stirred at room temperature for $6 \mathrm{~h}$. The reaction mixture was then diluted with EtOAc $(200 \mathrm{~mL})$ and the organic layer was washed with saturated aqueous $\mathrm{NaHCO}_{3}$ solution ( 5 x $75 \mathrm{~mL}$ ), dried over $\mathrm{MgSO}_{4}$ and concentrated in vacuo. The crude product (-)-15 was taken to the next step without purification.

Bis-TBS Protection: A $1 \mathrm{~L}$ round bottom flask was charged with crude keto diol (-)-15, $\mathrm{CH}_{2} \mathrm{Cl}_{2}(600$ $\mathrm{mL})$ and 2,6-lutidine $\left(12.7 \mathrm{~mL}, 109 \mathrm{mmol}, 4.8\right.$ equiv). The resulting solution was cooled to $0{ }^{\circ} \mathrm{C}$ then TBSOTf (10.45 mL, $45.5 \mathrm{mmol}, 2$ equiv) was added over 5 minutes. The reaction mixture was allowed to stir for $3.5 \mathrm{~h}$ at $0{ }^{\circ} \mathrm{C}$ then allowed to warm to room temperature. The reaction mixture was then poured into saturated aqueous $\mathrm{NH}_{4} \mathrm{Cl}$ solution $(500 \mathrm{~mL})$. The aqueous layer was extracted with $\mathrm{CH}_{2} \mathrm{Cl}_{2}$ $(2 \times 100 \mathrm{~mL})$ then the combined organic layers were washed with brine, dried over $\mathrm{MgSO}_{4}$ and concentrated in vacuo. The crude bis-TBS ether (-)-16 was preabsorbed onto silica gel (30 g) and subjected to column chromatography (Hexanes : $\mathrm{Et}_{2} \mathrm{O}=20: 1$ ) and the product was obtained as a white solid: $8.48 \mathrm{~g}(82 \%$ over three steps $) . \mathrm{mp}=97-98^{\circ} \mathrm{C} .[\alpha]_{\mathrm{D}}^{20}-5.6^{\circ}\left(c 0.94, \mathrm{CHCl}_{3}\right)$.

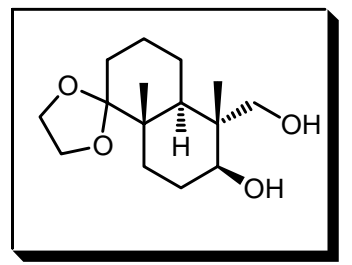

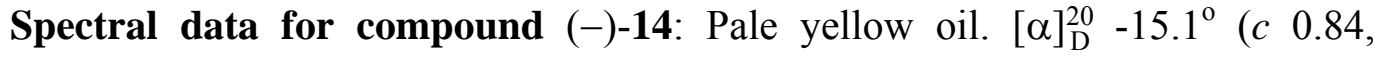
$\mathrm{CHCl}_{3}$ ); IR (neat) 3374.6 (s, br), 2944.5 (s), 2872.7 (s), 1453.8 (w), 1381.5 (w), $1200.2(\mathrm{w}), 1177.3(\mathrm{~m}), 1128.9(\mathrm{~m}), 1106.9(\mathrm{~m}), 1063.6(\mathrm{~m}), 1038.5(\mathrm{~s}), 906.4$ (w) $\mathrm{cm}^{-1} ;{ }^{1} \mathrm{H}$ NMR $\left(500 \mathrm{MHz}, \mathrm{CDCl}_{3}\right) \delta 0.90(\mathrm{~s} .3 \mathrm{H}), 1.07(\mathrm{~s}, 3 \mathrm{H}), 1.32-1.50(\mathrm{~m}, 6 \mathrm{H}), 1.52-1.68(\mathrm{~m}, 5$ H), 2.60 (br s, $2 \mathrm{H}), 3.43(\mathrm{~d}, J=10.4 \mathrm{~Hz}, 1 \mathrm{H}), 3.64(\mathrm{~m}, 1 \mathrm{H}), 3.67(\mathrm{~d}, J=10.4 \mathrm{~Hz}, 1 \mathrm{H}), 3.81(\mathrm{~m}, 1 \mathrm{H})$, 
$3.90(\mathrm{~m}, 3 \mathrm{H}) ;{ }^{13} \mathrm{C}$ NMR $\left(125 \mathrm{MHz}, \mathrm{CDCl}_{3}\right) \delta 113.1,77.1,72.3,65.3,64.8,43.1,42.91,41.9,30.3$ 28.4, 26.7, 22.9, 20.8, 16.9, 11.1. HRMS m/z 270.1837 [ $\mathrm{M}^{+}$; calcd for $\left.\mathrm{C}_{15} \mathrm{H}_{26} \mathrm{O}_{4}: 270.1831\right]$.

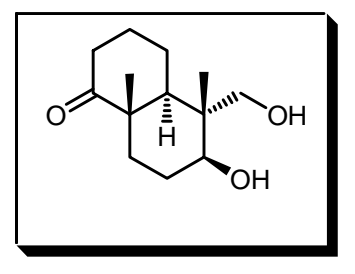

Spectral data for compound (-)-15: A white solid. $\mathrm{mp}=152-155{ }^{\circ} \mathrm{C}$. $[\alpha]_{\mathrm{D}}^{20}-43.5^{\mathrm{o}}$ (c 1.22, $\left.\mathrm{CHCl}_{3}\right)$; IR (film) $3471.5(\mathrm{~s}, \mathrm{br}), 3426.4(\mathrm{~s}, \mathrm{br}), 2933.9(\mathrm{~m})$, $2884.3(\mathrm{~m}), 1682.6(\mathrm{~s}), 1475.0(\mathrm{~m}), 1439.1(\mathrm{~m}), 1360.8(\mathrm{w}), 1343.7(\mathrm{w}), 1311.4$ (m), $1149.4(\mathrm{w}), 1109.8(\mathrm{~m}), 1093.9(\mathrm{w}), 1038.2(\mathrm{~m}), 998.5(\mathrm{~m}), 961.1(\mathrm{w}), 917.7(\mathrm{w}), 891.7(\mathrm{w}) \mathrm{cm}^{-1}$; ${ }^{1} \mathrm{H}$ NMR $\left(500 \mathrm{MHz}, \mathrm{CDCl}_{3}\right) \delta 0.92(\mathrm{~s}, 3 \mathrm{H}), 1.14(\mathrm{~s}, 3 \mathrm{H}), 1.31(\mathrm{dd}, J=2.9,12.3 \mathrm{~Hz}, 1 \mathrm{H}), 1.49-1.74$ (m, $7 \mathrm{H}), 2.03(\mathrm{~m}, 1 \mathrm{H}), 2.18(\mathrm{dddd}, J=1.7,1.7,4.5,14.2 \mathrm{~Hz}, 1 \mathrm{H}), 2.53(\mathrm{dt}, J=6.9,14.0 \mathrm{~Hz}, 1 \mathrm{H})$, 2.89 (br s, $2 \mathrm{H}), 3.36(\mathrm{~d}, J=10.5 \mathrm{~Hz}, 1 \mathrm{H}), 3.58(\mathrm{~m}, 1 \mathrm{H}), 3.65(\mathrm{~d}, J=10.5 \mathrm{~Hz}, 1 \mathrm{H}) ;{ }^{13} \mathrm{C}$ NMR $(125$ $\left.\mathrm{MHz}, \mathrm{CDCl}_{3}\right) \delta 215.1,75.1,70.2,48.2,46.7,42.9,37.4,31.0,26.4,25.9,20.7,18.9,11.7 . \mathrm{HRMS} \mathrm{m} / \mathrm{z}$ 226.1572 [M ; calcd. for $\mathrm{C}_{13} \mathrm{H}_{22} \mathrm{O}_{3}$ : 226.1569].

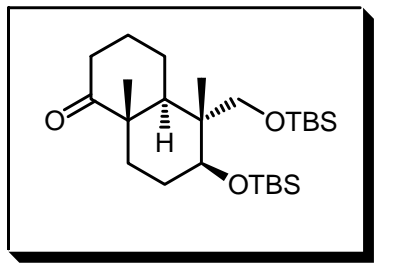

Spectral data for compound (-)-16: A white solid. $\mathrm{mp}=97-98{ }^{\circ} \mathrm{C}$. $[\alpha]_{\mathrm{D}}^{20}-5.6^{\mathrm{o}}\left(\mathrm{c} 0.94, \mathrm{CHCl}_{3}\right) . \mathrm{IR}($ film) $2935.9(\mathrm{~s}), 2855.8(\mathrm{~s}), 1698.7(\mathrm{~s}), 1472.1$ (m), $1385.4(\mathrm{w}), 1252.3(\mathrm{~m}), 1097.8(\mathrm{~s}), 1005.5(\mathrm{w}), 899.9(\mathrm{w}), 884.7(\mathrm{~m})$, 834.8 (s), $773.6(\mathrm{~s}) \mathrm{cm}^{-1} ;{ }^{1} \mathrm{H} \mathrm{NMR}\left(500 \mathrm{MHz}, \mathrm{CDCl}_{3}\right) \delta-0.03(\mathrm{~s}, 3 \mathrm{H}),-0.01(\mathrm{~s}, 3 \mathrm{H}), 0.00(\mathrm{~s}, 3 \mathrm{H}), 0.02$ (s, $3 \mathrm{H}), 0.68(\mathrm{~s}, 3 \mathrm{H}), 0.83(\mathrm{~s}, 9 \mathrm{H}), 0.84(\mathrm{~s}, 9 \mathrm{H}), 1.12(\mathrm{~s}, 3 \mathrm{H}), 1.40-1.66(\mathrm{~m}, 8 \mathrm{H}), 2.01(\mathrm{~m}, 1 \mathrm{H}), 2.16$ (m, $1 \mathrm{H}), 2.52(\mathrm{dt}, J=6.9,13.9 \mathrm{~Hz}, 1 \mathrm{H}), 3.12(\mathrm{~d}, J=9.8 \mathrm{~Hz}, 1 \mathrm{H}), 3.40(\mathrm{~d}, J=9.8 \mathrm{~Hz}, 1 \mathrm{H}), 3.63(\mathrm{dd}, J$ $=4.7,10.6 \mathrm{~Hz}, 1 \mathrm{H}) ;{ }^{13} \mathrm{C} \mathrm{NMR}\left(125 \mathrm{MHz}, \mathrm{CDCl}_{3}\right) \delta 215.6,71.2,64.2,48.1,44.4,37.5,30.9,27.0,26.1$, 
25.9, 25.8, 20.5, 18.8, 18.1 18.0, 12.9, -3.6, -4.9, -5.3, -5.8. HRMS (ESI) m/z 477.3215 [(M+Na) ${ }^{+}$; calcd. for $\mathrm{C}_{25} \mathrm{H}_{50} \mathrm{O}_{3} \mathrm{NaSi}_{2}$ : 477.3196$]$.

\section{Preparation of (4aS,5R,6S,8aS)-6-(tert-butyldimethylsilyloxy)-5-((tert-butyl-dimethylsilyloxy)} methyl)-5,8a-dimethyl-3,4,4a,5,6,7,8,8a-octahydronaphthalen-1-yl trifluoromethanesulfonate (-)17.

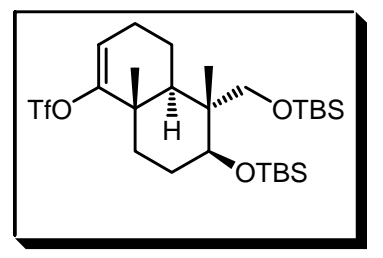

A $1 \mathrm{~L}$ round bottom flask was charged with bis-TBS ether (-)-16 (4.90 g, 10.77 mmol, 1 equiv), THF (359 mL). The resulting $0.03 \mathrm{M}$ solution was cooled to $78^{\circ} \mathrm{C}$ using an acetone/dry ice bath. Next, the $1.0 \mathrm{M}$ THF solution of LiHMDS ( $21.54 \mathrm{~mL}, 21.54 \mathrm{mmol}, 2$ equiv) was added over 10 minutes. The resulting solution was stirred at -78 ${ }^{\circ} \mathrm{C}$ for 25 minutes then a $0.5 \mathrm{M}$ solution of Comins' reagent $(7.91 \mathrm{~g}, 20.14 \mathrm{mmol}, 1.87$ equiv) in THF $(41 \mathrm{~mL})$ was added over 5 minutes. The reaction mixture then was allowed to slowly warm to room temperature over $2 \mathrm{~h}$. The progress of the reaction was monitored by TLC (Hexanes : EtOAc $=5: 1, R_{f}=$ 0.85). The reaction mixture was next diluted with $200 \mathrm{~mL}$ of saturated aqueous $\mathrm{NH}_{4} \mathrm{Cl}$ solution $(200$ $\mathrm{mL})$. The aqueous phase was extracted with EtOAc $(3 \times 150 \mathrm{~mL})$. The combined organic layers were washed with brine $(2 \times 150 \mathrm{~mL})$, dried over $\mathrm{MgSO}_{4}$ and concentrated in vacuo. The crude enol triflate product was preabsorbed on silica gel $(20 \mathrm{~g})$ and subjected to column chromatography (Hexanes : $\mathrm{Et}_{2} \mathrm{O}$ $=50: 1)$ and (-)-17 was obtained as a thick colorless oil: $5.70 \mathrm{~g}(90 \%) .[\alpha]_{\mathrm{D}}^{20}-32.6^{\circ}\left(c \quad 0.53, \mathrm{CHCl}_{3}\right)$;

IR (neat) 2953.9 (s, br), $1677.8(\mathrm{~m}), 1471.2(\mathrm{~m}), 1418.4(\mathrm{~s}), 1360.3(\mathrm{~m}), 1211.6(\mathrm{~s}), 1143.1(\mathrm{~s}), 986.2$ (m), $939.9(\mathrm{~m}), 921.3(\mathrm{~m}), 848.0(\mathrm{~m}), 774.5(\mathrm{~m}), 724.6(\mathrm{w}) \mathrm{cm}^{-1} ;{ }^{1} \mathrm{H} \mathrm{NMR}\left(500 \mathrm{MHz}, \mathrm{CDCl}_{3}\right) \delta 0.01(\mathrm{~s}$, $3 \mathrm{H}), 0.029$ (s, $3 \mathrm{H}), 0.034$ (s, $3 \mathrm{H}), 0.04(\mathrm{~s}, 3 \mathrm{H}), 0.62(\mathrm{~s}, 3 \mathrm{H}), 0.86(\mathrm{~s}, 9 \mathrm{H}), 0.88(\mathrm{~s}, 9 \mathrm{H}), 1.16(\mathrm{~s}, 3 \mathrm{H})$, 
1.30-1.44 (m, 2 H), 1.56-1.75 (m, 4 H), 1.78 (dd, $J=1.5,12.3 \mathrm{~Hz}, 1 \mathrm{H}), 2.10$ (m, $1 \mathrm{H}), 2.19$ (ddd, $J=$ 5.1, 5.1, $18.0 \mathrm{~Hz}, 1 \mathrm{H}), 3.17(\mathrm{~d}, J=9.8 \mathrm{~Hz}, 1 \mathrm{H}), 3.42$ (d, $J=9.8 \mathrm{~Hz}, 1 \mathrm{H}), 3.75(\mathrm{dd}, J=4.9,11.4 \mathrm{~Hz}, 1$ H), $5.54(\mathrm{dd}, J=2.7,5.0 \mathrm{~Hz}, 1 \mathrm{H}) ;{ }^{13} \mathrm{C} \mathrm{NMR}\left(125 \mathrm{MHz}, \mathrm{CDCl}_{3}\right) \delta 157.1,118.4(\mathrm{q}, J=312.5 \mathrm{~Hz})$, $114.6,71.2,64.3,43.3,43.0,38.7,32.4,29.7,27.1,25.9,24.5,19.6,18.1,17.4,12.7,-3.6,-4.9,-5.3,-$ 5.8. HRMS (ESI) $\mathrm{m} / \mathrm{z}$ 609.2681 [(M+Na) ${ }^{+}$; calcd. for $\mathrm{C}_{26} \mathrm{H}_{49} \mathrm{O}_{5} \mathrm{~F}_{3} \mathrm{NaSi}_{2} \mathrm{~S}_{1}$ : 609.2689].

Preparation of (4aR,5R,6S,8aS)-6-(tert-butyldimethylsilyloxy)-5-((tert-butyldimethylsilyloxy) methyl)-5,8a-dimethyl-3,4,4a,5,6,7,8,8a-octahydronaphthalene-1-carbaldehyde [(+)-18].

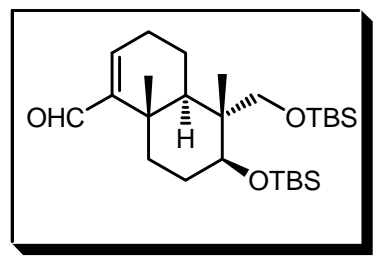

A $500 \mathrm{~mL}$ three-necked flask was charged with $\mathrm{LiCl}(1.79 \mathrm{~g}, 42.23 \mathrm{mmol}, 2.5$ equiv) that was dried overnight at $140{ }^{\circ} \mathrm{C}$ under vacuum, enol triflate $(-)-\mathbf{1 7}$ (9.93 g, 16.92 mmol, 1 equiv) and THF (174 mL). Next, carbon monoxide (CO) gas was bubbled through the solution for 10 minutes then $\mathrm{Pd}\left(\mathrm{PPh}_{3}\right)_{4}(1.95 \mathrm{~g}, 1.69 \mathrm{mmol}, 0.1$ equiv) was added in one portion. In the meantime $\mathrm{Bu}_{3} \mathrm{SnH}(5.88 \mathrm{~g}, 20.31 \mathrm{mmol}, 1.2$ equiv) was dissolved in THF (93 mL). While the CO gas was continuously bubbled through the reaction mixture, the temperature was raised to $50^{\circ} \mathrm{C}$ and the addition of the $\mathrm{Bu}_{3} \mathrm{SnH}$ solution via the syringe pump was initiated and was completed in $2 \mathrm{~h}$. The reaction mixture was then allowed to cool to room temperature and the solvent concentrated in vacuo. The crude product was preabsorbed on silica gel (25 g) and subjected to column chromatography (Hexanes : $\mathrm{Et}_{2} \mathrm{O}=75: 1$ ) and the product was obtained as a white crystalline solid: $7.21 \mathrm{~g}(92 \%) \cdot \mathrm{mp}=114-116^{\circ} \mathrm{C} .[\alpha]_{\mathrm{D}}^{20}+39.2^{\circ}\left(\mathrm{c} 1.43, \mathrm{CHCl}_{3}\right)$.

IR (film) 2935.9 (s), $2855.8(\mathrm{~m}), 1698.7(\mathrm{~s}), 1472.1(\mathrm{~m}), 1385.4(\mathrm{w}), 1252.3(\mathrm{~m}), 1097.8(\mathrm{~s}), 1005.5(\mathrm{w})$, 899.9 (w), 884.7 (m), 834.8 (s), 773.6 (s) cm ${ }^{-1} ;{ }^{1} \mathrm{H}$ NMR (500 MHz, CDCl$\left.{ }_{3}\right) \delta 0.01$ (s, $\left.6 \mathrm{H}\right), 0.02$ (s, 3 
H), $0.03(\mathrm{~s}, 3 \mathrm{H}), 0.62(\mathrm{~s}, 3 \mathrm{H}), 0.85(\mathrm{~s}, 9 \mathrm{H}), 0.86(\mathrm{~s}, 9 \mathrm{H}), 1.08(\mathrm{dt}, J=4.1,13.4 \mathrm{~Hz}, 1 \mathrm{H}), 1.14(\mathrm{~s}, 3 \mathrm{H})$, $1.39(\mathrm{~m}, 1 \mathrm{H}), 1.55-1.71(\mathrm{~m}, 4 \mathrm{H}), 2.27(\mathrm{~m}, 1 \mathrm{H}), 2.41(\mathrm{ddd}, J=4.9,4.9,20.4 \mathrm{~Hz}, 1 \mathrm{H}), 2.61(\mathrm{ddd}, J=$ 3.6, 3.6, 13.5 Hz, $1 \mathrm{H}), 3.12(\mathrm{~d}, J=9.8 \mathrm{~Hz}, 1 \mathrm{H}), 3.42$ (d, $J=9.8 \mathrm{~Hz}, 1 \mathrm{H}), 3.72(\mathrm{dd}, J=5.3,11.3 \mathrm{~Hz}, 1$ $\mathrm{H}), 6.54(\mathrm{dd}, J=2.8,4.5 \mathrm{~Hz}, 1 \mathrm{H}), 9.27(\mathrm{~s}, 1 \mathrm{H}) ;{ }^{13} \mathrm{C} \mathrm{NMR}\left(125 \mathrm{MHz}, \mathrm{CDCl}_{3}\right) \delta 194.1,152.4,151.5$, $71.5,64.3,43.5,42.6,36.7,33.0,28.4,27.5,25.92,20.1,18.1,18.1,17.3,12.8,-3.5,-4.9,-5.3,-5.8$; HRMS (ESI) $m / z 489.3177$ [(M+Na) ${ }^{+}$; calcd. for $\left.\mathrm{C}_{26} \mathrm{H}_{50} \mathrm{NaO}_{3} \mathrm{Si}_{2} ; 489.3196\right]$.

\section{Preparation of (S)-tert-butyl 2-amino-3,3-dimethylbutanoate [(+)-23].}

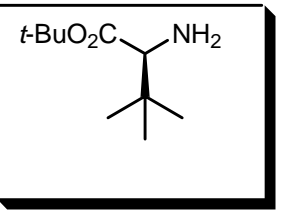

A large pressure tube $(150 \mathrm{~mL})$ was charged with L-tert-leucine $(+)-22(5.72 \mathrm{~g}$, $43.61 \mathrm{mmol}, 1$ equiv), dioxane $(30 \mathrm{~mL})$ and $\mathrm{CH}_{2} \mathrm{Cl}_{2}(30 \mathrm{~mL})$. The resulting suspension was cooled to $0{ }^{\circ} \mathrm{C}$ then concentrated $\mathrm{H}_{2} \mathrm{SO}_{4}(2.6 \mathrm{~mL}, 48.5 \mathrm{mmol}, 1.11$ equiv) was added immediately turning the color of the suspension yellow. Next, the pressure tube was cooled to ca. $-30{ }^{\circ} \mathrm{C}$ and 2-methyl-2-propene $(30 \mathrm{~mL}, 300 \mathrm{mmol}, 6.9$ equiv) was added. The tube was then sealed and allowed to warm to room temperature. (Warning: the reaction should be kept behind a blast shield.) The reaction mixture was stirred for $23 \mathrm{~h}$ at room temperature at which point it became almost colorless and homogeneous. The pressure tube was next cooled to ca. $-30{ }^{\circ} \mathrm{C}$, slowly opened (only a gently hiss was audible) and the solvents were concentrated in vacuo. The pale yellow residue was then taken up in $10 \% \mathrm{NaOH}(100 \mathrm{~mL})$ and diluted with water so that the $\mathrm{pH}$ was $\sim 11$. The resulting mixture was extracted with $\mathrm{CH}_{2} \mathrm{Cl}_{2}(3 \times 100 \mathrm{~mL})$ and the combined organic layers were washed with water $(100 \mathrm{~mL})$, brine $(100 \mathrm{~mL})$, dried over $\mathrm{Na}_{2} \mathrm{SO}_{4}$ and concentrated in vacuo.(Remark: the bath temperature should be kept $\leq 30{ }^{\circ} \mathrm{C}$ to avoid losses of the product.) The crude product was purified by vacuum distillation and the product was obtained as a pale yellow oil: $6.7 \mathrm{~g}(83 \%)$. $[\alpha]_{\mathrm{D}}^{20}+58.6^{\circ}(\mathrm{c} 1.16$, benzene). 
IR (neat) 3391.9 (w, br), 2976.1 (s), $2871.5(\mathrm{~m}), 1725.0$ (s), $1597.3(\mathrm{w}), 1478.9(\mathrm{~m}), 1392.8(\mathrm{~m}), 1367.5$ (s), $1320.5(\mathrm{w}), 1256.2(\mathrm{~m}), 1150.8(\mathrm{~s}), 1031.5(\mathrm{w}), 965.9(\mathrm{~m}), 932.7(\mathrm{w}), 848.7(\mathrm{~m}) \mathrm{cm}^{-1} ;{ }^{1} \mathrm{H}$ NMR (500 $\left.\mathrm{MHz}, \mathrm{CDCl}_{3}\right) \delta 0.95(\mathrm{~s}, 9 \mathrm{H}), 1.35($ br s, $2 \mathrm{H}), 1.45(\mathrm{~s}, 9 \mathrm{H}), 3.00(\mathrm{~s}, 1 \mathrm{H}) ;{ }^{13} \mathrm{C} \mathrm{NMR}\left(125 \mathrm{MHz}, \mathrm{CDCl}_{3}\right)$ $\delta$ 174.3, 80.8, 64.0, 34.4, 28.2, 26.4. HRMS (CI-MS) m/z 188.1650 [(M+H) ${ }^{+}$; calcd. for $\mathrm{C}_{10} \mathrm{H}_{22} \mathrm{~N}_{1} \mathrm{O}_{2}$ : 188.1650].

\author{
Preparation of (S)-tert-butyl 2-((E)-((4aR,5R,6S,8aS)-6-(tert-butyldimethylsilyloxy)-5-((tert- \\ butyldimethylsilyloxy)methyl)-5,8a-dimethyl-3,4,4a,5,6,7,8,8a-octahydronaphthalen-1-yl) \\ methyleneamino)-3,3-dimethylbutanoate [(+)-24].
}

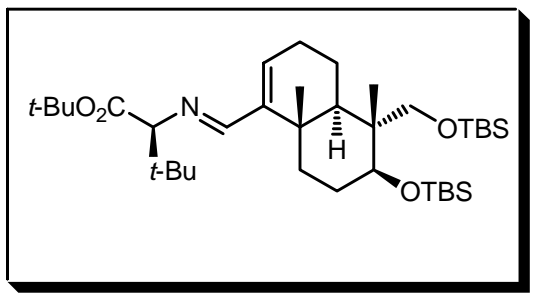

A $500 \mathrm{~mL}$ round bottom flask was charged with $(+)-23(3.22 \mathrm{~g}, 17.19$ mmol, 1.25 equiv) and toluene $(100 \mathrm{~mL})$ and $37 \%$ aqueous $\mathrm{HCl}(0.3$ $\mathrm{mL})$. The resulting mixture was then concentrated on the rotavap to a residue (bath temperature was $46^{\circ} \mathrm{C}$, pressure was 100 Torr). Then $\alpha, \beta$-unsaturated aldehyde $(+)-18(6.42 \mathrm{~g}, 13.75 \mathrm{mmol}, 1 \mathrm{equiv})$ and toluene $(100 \mathrm{~mL})$ were added and the process was repeated. After four cycles the progress of the reaction was checked by ${ }^{1} \mathrm{H}-\mathrm{NMR}\left(\mathrm{C}_{6} \mathrm{D}_{6}\right)$ indicating that $\geq 95 \%$ of the starting material was consumed. The crude product was loaded onto a flash column with basic alumina using Hexanes : $\mathrm{Et}_{2} \mathrm{O}=50: 1$ as the eluent and the product was isolated as a white solid: $8.5 \mathrm{~g}(97 \%)$. $[\alpha]_{\mathrm{D}}^{20}+39.4^{\circ}$ (c 2.17, benzene).

IR (neat) $2956.2(\mathrm{~s}, \mathrm{br}), 1716.7(\mathrm{~s}), 1634.4(\mathrm{~s}), 1472.9(\mathrm{~s}), 1387.6(\mathrm{~m}), 1368.0(\mathrm{~m}), 1253.9(\mathrm{~m}), 1102.5$ (m), 998.0 (m), $938.6(\mathrm{~m}), 890.6(\mathrm{~m}), 836.1(\mathrm{~m}), 769.6(\mathrm{~m}) ;{ }^{1} \mathrm{H}$ NMR (500 MHz, $\left.\mathrm{C}_{6} \mathrm{D}_{6}\right) \delta 0.03(\mathrm{~s}, 3 \mathrm{H})$, 0.06 (s, 3 H), 0.08 (s, 3 H), $0.12(\mathrm{~s}, 3 \mathrm{H}), 0.76$ (s, $3 \mathrm{H}), 0.96$ (s, $9 \mathrm{H}), 0.99$ (s, 9 H), 1.12 (s, $9 \mathrm{H}), 1.27-$ 
1.38 (m, 2 H), 1.39 (s, 9 H), 1.49 (s, 3 H), $1.52(\mathrm{~m}, 1 \mathrm{H}), 1.76-1.92$ (m, $3 \mathrm{H}), 2.04$ (ddd, J = 4.9, 4.9, 19.4 Hz, $1 \mathrm{H}), 2.19$ (m, $1 \mathrm{H}), 2.94(\mathrm{~m}, 1 \mathrm{H}), 3.22$ (d, J=9.7 Hz, $1 \mathrm{H}), 3.35$ (ddd, J= 3,5, 3.5, 13.4 Hz, 1 H), $3.37(\mathrm{~s}, 1 \mathrm{H}), 3.55(\mathrm{~d}, J=9.8 \mathrm{~Hz}, 1 \mathrm{H}), 3.94(\mathrm{dd}, J=5.1,11.6 \mathrm{~Hz}, 1 \mathrm{H}), 5.73(\mathrm{dd}, J=2.6,4.8 \mathrm{~Hz}, 1$ H), $7.53(\mathrm{~s}, 1 \mathrm{H}) ;{ }^{13} \mathrm{C} \mathrm{NMR}\left(125 \mathrm{MHz}, \mathrm{C}_{6} \mathrm{D}_{6}\right) \delta 170.6,164.6,148.3,139.8,85.0,80.0,72.2,64.9,43.9$, 43.7, 38.3, 34.9, 34.4, 28.4, 28.3, 28.2, 27.3, 26.2, 26.1, 19.9, 18.4, 18.3, 17.9, 13.4, -3.4, -4.7, -5.1, -5.7. HRMS (ESI) $m / z 658.4645\left[(\mathrm{M}+\mathrm{Na})^{+}\right.$; calcd. for $\mathrm{C}_{36} \mathrm{H}_{69} \mathrm{~N}_{1} \mathrm{O}_{4} \mathrm{NaSi}_{2}$ : 658.4663].

\section{Preparation of (1S,2R,4aR,5R,6S,8aS)-6-(tert-butyldimethylsilyloxy)-5-((tert-butyldimethyl-} silyloxy)methyl)-1,5,8a-trimethyl-2-vinyldecahydronaphthalene-1-carbaldehyde [(+)-25].

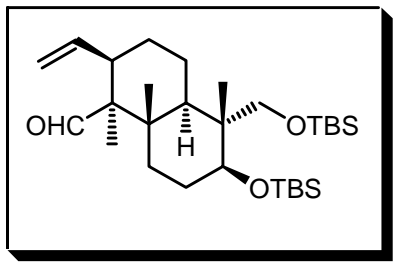

The Koga Reaction: A 100 mL Schlenk flask was charged with aldimine (+)24 (2.50 g, $3.93 \mathrm{mmol}, 1$ equiv) and THF $(27 \mathrm{~mL})$. The resulting solution was cooled to $-10{ }^{\circ} \mathrm{C}$ and $1.0 \mathrm{M}$ THF solution of vinylmagnesium bromide (23.6

$\mathrm{mL}, 23.6 \mathrm{mmol}, 6$ equiv) was added over 15 minutes. The resulting solution was then stirred for 90 minutes at $-10{ }^{\circ} \mathrm{C}$ during which time the solution became cloudy. Next, neat HMPA (5.3 mL, 30.5 mmol, 7.7 equiv) and neat MeI (5.3 mL, $84 \mathrm{mmol}, 22$ equiv) were added sequentially to the reaction mixture and the temperature was kept at $-10{ }^{\circ} \mathrm{C}$ for $3 \mathrm{~h}$ then it was allowed to warm to room temperature where it was stirred for $21 \mathrm{~h}$. The progress of the reaction was monitored by TLC (Hexanes : $\mathrm{Et}_{2} \mathrm{O}=$ 10:1, $\left.\mathrm{R}_{\mathrm{f}}=0.75\right)$. The reaction mixture was then diluted with $\mathrm{Et}_{2} \mathrm{O}(100 \mathrm{~mL})$, then distilled water was added $(50 \mathrm{~mL})$. Next, the aqueous phase was extracted with $\mathrm{Et}_{2} \mathrm{O}(3 \times 50 \mathrm{~mL})$ and the combined organic layers were washed first with $10 \%$ aqueous $\mathrm{Na}_{2} \mathrm{~S}_{2} \mathrm{O}_{3}$ solution $(25 \mathrm{~mL})$ then with brine $(2 \times 50 \mathrm{~mL})$, dried over $\mathrm{MgSO}_{4}$ and concentrated in vacuo to afford approximately $2.85 \mathrm{~g}$ of crude product that was taken to the hydrolysis step without purification. 
Imine Hydrolysis: The crude product $(\sim 2.85 \mathrm{~g})$ was dissolved in $\mathrm{CH}_{2} \mathrm{Cl}_{2}(35 \mathrm{~mL})$ then $10 \%$ aqueous solution of oxalic acid was added $(105 \mathrm{~mL})$. The resulting biphasic mixture was stirred vigorously for $12 \mathrm{~h}$. The reaction mixture was then diluted with $\mathrm{CH}_{2} \mathrm{Cl}_{2}(50 \mathrm{~mL})$. The organic phase was washed with water $(2 \times 50 \mathrm{~mL})$ and saturated aqueous $\mathrm{NaHCO}_{3}$ solution $(2 \times 50 \mathrm{~mL})$ then dried over $\mathrm{MgSO}_{4}$ and concentrated in vacuo. The crude vinyl aldehyde was subjected to column chromatography (Hexanes : $\left.\mathrm{Et}_{2} \mathrm{O}=50: 1\right)$ and the product was obtained as a white crystalline solid: $1.47 \mathrm{~g}(73 \%) . \mathrm{mp}=119-122^{\circ} \mathrm{C}$. $[\alpha]_{\mathrm{D}}^{20}+32.7^{\mathrm{o}}\left(\right.$ c $\left.0.87, \mathrm{C}_{6} \mathrm{D}_{6}\right)$

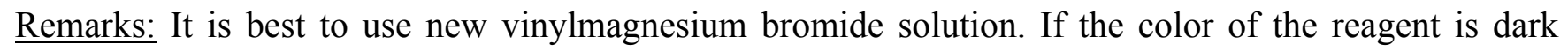
brown, it is usually indicative of poor quality for the purposes of the Koga reaction.

IR (film) 2926.4 (s), 2861.7 (m), 1716.7 (m), 1471.4 (m), 1386.9 (w), 1248.8 (m), 1086.3 (s), 834.8 (s), $772.1(\mathrm{~s}) ;{ }^{1} \mathrm{H} \mathrm{NMR}\left(500 \mathrm{MHz}, \mathrm{CDCl}_{3}\right) \delta 0.01(\mathrm{~s}, 3 \mathrm{H}), 0.07(\mathrm{~s}, 3 \mathrm{H}), 0.11(\mathrm{~s}, 3 \mathrm{H}), 0.13(\mathrm{~s}, 3 \mathrm{H}), 0.66(\mathrm{~s}$, $3 \mathrm{H}), 0.99(\mathrm{~s}, 9 \mathrm{H}), 1.01(\mathrm{~s}, 9 \mathrm{H}), 1.07(\mathrm{~s}, 3 \mathrm{H}), 1.13(\mathrm{~s}, 3 \mathrm{H}), 1.17-1.42(\mathrm{~m}, 3 \mathrm{H}), 1.49$ (m, $1 \mathrm{H}), 1.61-$ $1.72(\mathrm{~m}, 4 \mathrm{H}), 1.78(\mathrm{dd}, J=3.6,12.9 \mathrm{~Hz}, 1 \mathrm{H}), 2.39(\mathrm{~m}, 1 \mathrm{H}), 3.09$ (d, $J=9.8 \mathrm{~Hz}, 1 \mathrm{H}), 3.46(\mathrm{~d}, J=9.8$ Hz, $1 \mathrm{H}), 3.76$ (dd, $J=4.9,12.0 \mathrm{~Hz}, 1 \mathrm{H}), 4.96$ (ddd, $J=1.8,1.8,10.6 \mathrm{~Hz}, 1 \mathrm{H}), 5.00$ (ddd, $J=1.8,1.8$, 17.2 Hz, $1 \mathrm{H}), 6.12(\mathrm{ddd}, J=10.7,6.6,17.2 \mathrm{~Hz}, 1 \mathrm{H}), 9.64(\mathrm{~s}, 1 \mathrm{H}) ;{ }^{13} \mathrm{C} \mathrm{NMR}\left(125 \mathrm{MHz}, \mathrm{C}_{6} \mathrm{D}_{6}\right) \delta 204.5$, $140.9,114.3,71.9,64.5,55.8,45.9,44.0,39.0,38.4,32.2,27.3,27.2,26.2,26.2,26.1,25.7,19.3,18.9$, 18.4, 18.2, 17.8, 13.0, -3.4, -4.7, -5.2, -5.7. HRMS (ESI) $\mathrm{m} / \mathrm{z} 531.3671\left[(\mathrm{M}+\mathrm{Na})^{+}\right.$; calcd. for $\left.\mathrm{C}_{29} \mathrm{H}_{56} \mathrm{O}_{3} \mathrm{NaSi}_{2}: 531.3666\right]$. 


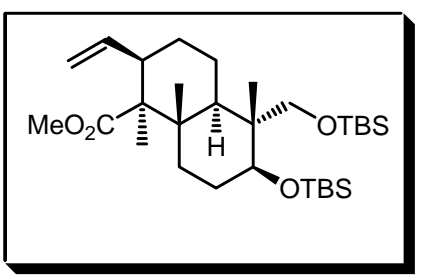

Pinnick Oxidation: A $500 \mathrm{~mL}$ round bottom flask was charged with vinyl aldehyde (+)-25 (2.38 g, $4.68 \mathrm{mmol}, 1$ equiv), $t-\mathrm{BuOH}(130 \mathrm{~mL})$ and a $2.0 \mathrm{M}$ THF solution of $99 \%+$ redistilled quality 2-methyl-2-butene $(9.18 \mathrm{~mL}$ alkene, $87 \mathrm{mmol}, 19$ equiv dissolved in $65 \mathrm{~mL}$ THF). The resulting solution was cooled to $0{ }^{\circ} \mathrm{C}$ with an ice bath and the aqueous solution of $\mathrm{NaH}_{2} \mathrm{PO}_{4}\left(3.98 \mathrm{~g}, 33.17 \mathrm{mmol}, 7.1\right.$ equiv) and $\mathrm{NaClO}_{2}(3.75 \mathrm{~g}$, $33.17 \mathrm{mmol}, 7.1$ equiv, $80 \%$ purity) in water (68 mL, HPLC grade) was added over 10 minutes. The reaction mixture then turned light yellow and was allowed to warm to room temperature where it was stirred for 40 minutes. The reaction mixture was then quenched with saturated aqueous $\mathrm{NH}_{4} \mathrm{Cl}$ solution $(200 \mathrm{~mL})$ then extracted with EtOAc $(5 \times 100 \mathrm{~mL})$. The combined organic layers were washed with brine $(100 \mathrm{~mL})$, dried over $\mathrm{MgSO}_{4}$ and concentrated in vacuo. The crude product $(+)-\mathbf{2 6}$ was taken to the next step as is.

Methyl Ester Formation: A $250 \mathrm{~mL}$ round bottom flask was charged with the crude carboxylic acid (+)26 ( 2.46 g, $4.68 \mathrm{mmol}, 1$ equiv), $\mathrm{MeOH}(17 \mathrm{~mL})$ and benzene (62 mL). The resulting solution was treated with the 2.0 M solution of $\mathrm{TMSCHN}_{2}$ in $\mathrm{Et}_{2} \mathrm{O}(3.51 \mathrm{~mL}, 7.02 \mathrm{mmol}, 1.5$ equiv). The resulting yellow solution was allowed to stir for 30 minutes at room temperature. Next, the excess $\mathrm{TMSCHN}_{2}$ was destroyed with a few drops of glacial $\mathrm{AcOH}$ after which the yellow color of the reaction mixture dissipated. The solvents were then concentrated in vacuo to afford the crude methyl ester (+)-27. The crude product was subjected to column chromatography (Hexanes : $\mathrm{Et}_{2} \mathrm{O}=200: 1$ ) and the product was obtained as a white crystalline solid: $2.07 \mathrm{~g}(82 \%) . \mathrm{mp}=146-148^{\circ} \mathrm{C} .[\alpha]_{\mathrm{D}}^{20}+11.3^{\circ}\left(\mathrm{c} 0.63, \mathrm{CHCl}_{3}\right)$; 


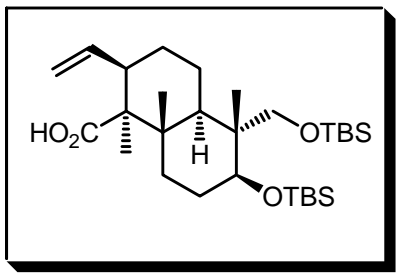

Spectral data for compound (+)-26: A white solid. $m p=232-235^{\circ} \mathrm{C} \cdot[\alpha]_{\mathrm{D}}^{20}$ $+21.8^{\circ}$ (c 1.05, $\left.\mathrm{CHCl}_{3}\right)$. IR (film) $2953.0(\mathrm{~s}, \mathrm{br}), 2929.3(\mathrm{~s}), 2859.0(\mathrm{~m})$, $1702.6(\mathrm{~s}), 1470.7(\mathrm{~m}), 1386.1(\mathrm{w}), 1249.9(\mathrm{~m}), 1085.5(\mathrm{~s}), 1005.0(\mathrm{w}), 905.9$ (w), 884.9 (w), $834.8(\mathrm{~m}), 772.4(\mathrm{~m}) \mathrm{cm}^{-1} ;{ }^{1} \mathrm{H}$ NMR $\left(500 \mathrm{MHz}, \mathrm{CDCl}_{3}\right) \delta 0.02(\mathrm{~s}, 3 \mathrm{H}), 0.02(\mathrm{~s}, 3 \mathrm{H})$, 0.03 (s, $6 \mathrm{H}), 0.59$ (s, $3 \mathrm{H}), 0.85$ (s, $9 \mathrm{H}), 0.91$ (s, $9 \mathrm{H}), 1.18$ (s, $3 \mathrm{H}), 1.20-1.37$ (m, $3 \mathrm{H}), 1.42$ (s, $3 \mathrm{H})$, 1.47 (m, 2 H), $1.62(\mathrm{~m}, 1 \mathrm{H}), 1.74(\mathrm{~m}, 1 \mathrm{H}), 1.85(\mathrm{~m}, 1 \mathrm{H}), 1.92$ (dd, $J=2.7,12.4 \mathrm{~Hz}, 1 \mathrm{H}), 2.07$ (ddd, $J$ $=3.4,3.4,13.3 \mathrm{~Hz}, 1 \mathrm{H}), 2.61(\mathrm{~m}, 1 \mathrm{H}), 3.11(\mathrm{~d}, J=9.7 \mathrm{~Hz}, 1 \mathrm{H}), 3.37(\mathrm{~d}, J=9.8 \mathrm{~Hz}, 1 \mathrm{H}), 3.64(\mathrm{dd}, J$ $=4.8,11.6 \mathrm{~Hz}, 1 \mathrm{H}), 4.96(\mathrm{ddd}, J=1.7,1.7,10.6 \mathrm{~Hz}, 1 \mathrm{H}), 5.04(\mathrm{ddd}, J=1.7,1.7,17.2 \mathrm{~Hz}, 1 \mathrm{H}), 6.10$ (ddd, $J=10.7,6.2,17.1 \mathrm{~Hz}, 1 \mathrm{H}) ;{ }^{13} \mathrm{C}$ NMR $\left(125 \mathrm{MHz}, \mathrm{CDCl}_{3}\right) \delta 181.9,141.6,113.3,71.7,64.3,55.3$, $46.6,43.8,39.5,37.7,31.9,27.2,26.0,25.9,25.9,24.5,22.8,19.1,18.1,18.0,17.3,12.8,-3.6,-4.9,-$ 5.3, -5.7. HRMS (ESI) $\mathrm{m} / \mathrm{z} 547.3589\left[(\mathrm{M}+\mathrm{Na})^{+}\right.$; calcd for $\left.\mathrm{C}_{29} \mathrm{H}_{56} \mathrm{O}_{4} \mathrm{NaSi}_{2}: 547.3615\right]$.

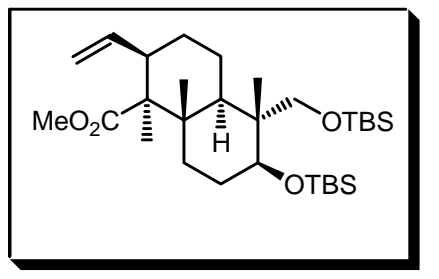

Spectral data for compound (+)-27: A white solid. $\mathrm{mp}=146-148{ }^{\circ} \mathrm{C}$. $[\alpha]_{\mathrm{D}}^{20}+11.3^{\circ}\left(c\right.$ 0.63, $\left.\mathrm{CHCl}_{3}\right)$; IR (film) $2951.3(\mathrm{~m}), 2925.7(\mathrm{~m}), 2856.5(\mathrm{~m})$, $1732.7(\mathrm{~s}), 1472.4(\mathrm{w}), 1387.3(\mathrm{w}), 1255.2(\mathrm{~m}), 1081.6(\mathrm{~s}), 1002.3(\mathrm{w})$, $917.0(\mathrm{~m}), 834.5(\mathrm{~m}), 770.9(\mathrm{~m}) \mathrm{cm}^{-1} ;{ }^{1} \mathrm{H}$ NMR $\left(500 \mathrm{MHz}, \mathrm{CDCl}_{3}\right) \delta 0.01(\mathrm{~s}, 3 \mathrm{H}), 0.02(\mathrm{~s}, 9 \mathrm{H}), 0.59(\mathrm{~s}$, $3 \mathrm{H}), 0.85$ (s, $9 \mathrm{H}), 0.91$ (s, $9 \mathrm{H}), 1.16(\mathrm{~s}, 3 \mathrm{H}), 1.24-1.32(\mathrm{~m}, 2 \mathrm{H}), 1.35(\mathrm{~s}, 3 \mathrm{H}), 1.43-1.51(\mathrm{~m}, 2 \mathrm{H})$, 1.61-1.81 (m, $3 \mathrm{H}), 1.91$ (dd, $J=2.8,12.4 \mathrm{~Hz}, 1 \mathrm{H}), 2.06$ (ddd, $J=3.4,3.4,13.3 \mathrm{~Hz}, 1 \mathrm{H}), 2.60$ (m, 1 H), $3.10(\mathrm{~d}, J=9.7 \mathrm{~Hz}, 1 \mathrm{H}), 3.36(\mathrm{~d}, J=9.7 \mathrm{~Hz}, 1 \mathrm{H}), 3.59(\mathrm{~s}, 3 \mathrm{H}), 3.64(\mathrm{dd}, J=4.7,11.7 \mathrm{~Hz}, 1 \mathrm{H})$, $4.92(\mathrm{~d}, J=10.6 \mathrm{~Hz}, 1 \mathrm{H}), 4.96(\mathrm{~d}, J=17.2 \mathrm{~Hz}, 1 \mathrm{H}), 6.00(\mathrm{ddd}, J=10.6,6.5,17.2 \mathrm{~Hz}, 1 \mathrm{H}) ;{ }^{13} \mathrm{C} \mathrm{NMR}$ $\left(125 \mathrm{MHz}, \mathrm{CDCl}_{3}\right) \delta 176.3,142.0,113.1,71.7,64.3,55.3,50.7,47.0,43.8,39.6,37.6,31.9,27.2,25.9$, 25.9, 25.9, 25.9, 24.7, 22.7, 19.1, 18.1, 18.0, 17.3, 12.8, -3.6, -4.9, -5.3, -5.7. HRMS (ESI) $\mathrm{m} / \mathrm{z} 561.3769$ $\left[(\mathrm{M}+\mathrm{Na})^{+}\right.$; calcd for $\left.\mathrm{C}_{30} \mathrm{H}_{58} \mathrm{O}_{4} \mathrm{NaSi}_{2}: 561.3771\right]$. 


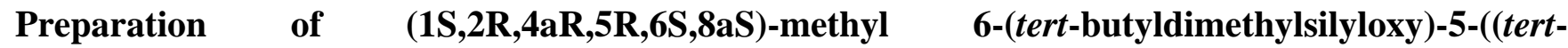
butyldimethylsilyloxy)methyl)-2-formyl-1,5,8a-trimethyldecahydronaphthalene-1-carboxylate $[(+)-28]$.

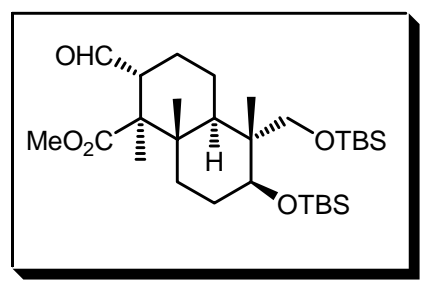

Ozonolysis: A $500 \mathrm{~mL}$ round bottom flask, equipped with a PTFE coated stir-bar, was charged with (+)-27 (1.69 g, 3.14 mmol, 1 equiv) and $\mathrm{CH}_{2} \mathrm{Cl}_{2}$ $(185 \mathrm{~mL})$. The resulting clear, colorless solution was cooled to $-78^{\circ} \mathrm{C}$ with an acetone/dry ice bath and treated with a stream of ozonized oxygen until the starting material was consumed (by TLC). The progress of the reaction was monitored by TLC (Hexanes : $\mathrm{Et}_{2} \mathrm{O}=9: 1$; developed with anisaldehyde). The starting material was consumed in 18 minutes after which the reaction mixture was purged for 15 minutes with a stream of argon to remove any residual ozone. The reaction mixture was next quenched with neat $\mathrm{Me}_{2} \mathrm{~S}\left(14 \mathrm{~mL}, 147 \mathrm{mmol}, 47\right.$ equiv) at $-78{ }^{\circ} \mathrm{C}$ and then warmed to room temperature and aged for 48 hours. Next, the solvent and the excess $\mathrm{Me}_{2} \mathrm{~S}$ were removed in vacuo. (Warning: the sterically hindered nature of the ozonide results in a very slow reaction rate, one must make sure that all the ozonide has been destroyed to avoid potential explosive decomposition on the rotary evaporator.) The crude aldehyde product $(+)-\mathbf{2 8}$ was taken to the next step without purification.

Isomerization of the C(12) Stereocenter: The crude aldehyde $(+)-28(\sim 1.6 \mathrm{~g})$ was dissolved in THF (21 $\mathrm{mL})$. To the resulting solution $\mathrm{DBU}(857 \mathrm{mg}, 842 \mu \mathrm{L}, 1.8$ equiv) was added at room temperature in one portion. The reaction mixture was stirred for $4 \mathrm{~h}$ at room temperature until the complete consumption of the starting material was indicated by TLC analysis (Hexanes : EtOAc $=5: 1$ ). The solvent was then removed in vacuo to afford the crude product that was subjected to column chromatography (Hexanes : $\left.\mathrm{Et}_{2} \mathrm{O}=20: 1\right)$. The product $(+)-29$ was obtained as a white solid: $1.38 \mathrm{~g}(59 \%$ for 2 steps $) . \mathrm{mp}=97-99$ ${ }^{\circ} \mathrm{C} ;[\alpha]_{\mathrm{D}}^{20}+5.0^{\circ}\left(c 0.44, \mathrm{CHCl}_{3}\right)$. 
IR (film) 2952.0, (s, br) 2857.0 (m), 1722.4 (s), $1471.2(\mathrm{~m}), 1446.8(\mathrm{~m}), 1389.0(\mathrm{~m}), 1359.8$ (w), 1250.6 (m), 1217.3 (w), 1158.0 (w), 1094.4 (m), 1004.5 (w), 938.2 (w), 836.2 (m), 774.0 (m) cm ${ }^{-1} ;{ }^{1} \mathrm{H}$ NMR $\left(500 \mathrm{MHz}, \mathrm{CDCl}_{3}\right) \delta 0.02(\mathrm{~s}, 3 \mathrm{H}), 0.03(\mathrm{~s}, 6 \mathrm{H}), 0.035(\mathrm{~s}, 3 \mathrm{H}), 0.61(\mathrm{~s}, 3 \mathrm{H}), 0.86(\mathrm{~s}, 9 \mathrm{H}), 0.89(\mathrm{~s}, 9$ H), $1.01(\mathrm{~s}, 3 \mathrm{H}), 1.21(\mathrm{~s}, 3 \mathrm{H}), 1.31-1.39(\mathrm{~m}, 3 \mathrm{H}), 1.51-1.60(\mathrm{~m}, 4 \mathrm{H}), 1.91(\mathrm{dd}, J=2.7,12.1 \mathrm{~Hz}, 1 \mathrm{H})$, $1.98(\mathrm{~m}, 1 \mathrm{H}), 3.11(\mathrm{~d}, J=9.8 \mathrm{~Hz}, 1 \mathrm{H}), 3.21(\mathrm{dd}, J=4.2,12.7 \mathrm{~Hz}, 1 \mathrm{H}), 3.37(\mathrm{~d}, J=9.9 \mathrm{~Hz}, 1 \mathrm{H}), 3.66$ $(\mathrm{dd}, J=2.1,8.7 \mathrm{~Hz}, 1 \mathrm{H}), 3.68(\mathrm{~s}, 3 \mathrm{H}), 9.57(\mathrm{~s}, 1 \mathrm{H}) ;{ }^{13} \mathrm{C} \mathrm{NMR}\left(125 \mathrm{MHz}, \mathrm{CDCl}_{3}\right) \delta 204.4,175.3,71.3$, 64.1, 52.5, 51.5, 50.8, 43.8, 39.7, 36.5, 31.2, 27.4, 25.9, 25.9, 25.8, 21.9, 20.5, 18.2, 17.9, 17.7, 13.5, 12.8, -3.7, -4.9, -5.4, -5.7. HRMS (ESI) $\mathrm{m} / \mathrm{z} 563.3585$ [(M+Na) ${ }^{+}$; calcd for $\left.\mathrm{C}_{29} \mathrm{H}_{56} \mathrm{O}_{5} \mathrm{NaSi}_{2}: 563.3564\right]$.

Preparation of $\quad(1 \mathrm{~S}, 2 \mathrm{R}, 4 \mathrm{aR}, 5 \mathrm{R}, 6 \mathrm{~S}, 8 \mathrm{aS})$-methyl 6-(tert-butyldimethylsilyloxy)-5-((tertbutyldimethylsilyloxy)methyl)-2-(hydroxymethyl)-1,5,8a-trimethyldecahydronaphthalene-1carboxylate $[(+)-30]$.

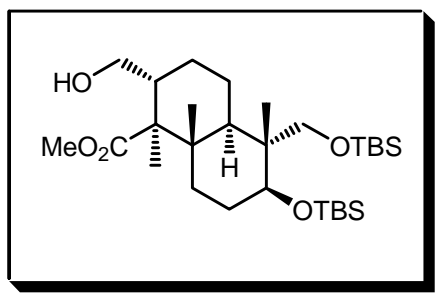

A $200 \mathrm{~mL}$ round bottom flask was charged with inverted aldehyde $(+)-\mathbf{2 9}$ (500 mg, $0.924 \mathrm{mmol}, 1$ equiv) and $\mathrm{MeOH}(80 \mathrm{~mL})$. The resulting solution was cooled to $0{ }^{\circ} \mathrm{C}$ with an ice-water bath. Next, $\mathrm{NaBH}_{4}(325 \mathrm{mg}, 8.6$ mmol, 9.3 equiv) was added in one portion. The reaction mixture was then allowed to warm to room temperature in 30 minutes. The reaction was then quenched with brine (30 $\mathrm{mL})$ then the $\mathrm{MeOH}$ was removed in vacuo to afford a white slurry that was extracted with EtOAc $(5 \mathrm{x}$ $60 \mathrm{~mL}$ ). The combined organic layers were dried over $\mathrm{MgSO}_{4}$ and concentrated in vacuo to afford analytically pure product that was obtained as a white crystalline residue: $540 \mathrm{mg}(99 \%)$. $\mathrm{mp}=126-129$ ${ }^{\circ} \mathrm{C} .[\alpha]_{\mathrm{D}}^{20}+7.0^{\circ}\left(\right.$ c $\left.2.1, \mathrm{CHCl}_{3}\right)$. 
IR (film) 3507.2 (w, br), 2952.7 (s), 2857.0 (m), 1722.4 (m), $1472.4(\mathrm{~m}), 1388.5$ (m), 1360.5 (w), 1251.1 (m), 1159.5 (w), $1090.3(\mathrm{~m}), 1006.4(\mathrm{~m}), 938.9(\mathrm{w}), 890.2(\mathrm{w}), 835.3(\mathrm{~m}), 774.0(\mathrm{~m}) \mathrm{cm}^{-1} ;{ }^{1} \mathrm{H}$ $\operatorname{NMR}\left(500 \mathrm{MHz}, \mathrm{C}_{6} \mathrm{D}_{6}\right) \delta 0.01(\mathrm{~s}, 3 \mathrm{H}), 0.07(\mathrm{~s}, 3 \mathrm{H}), 0.12(\mathrm{~s}, 3 \mathrm{H}), 0.14(\mathrm{~s}, 3 \mathrm{H}), 0.70(\mathrm{~s}, 3 \mathrm{H}), 0.99$ (s, $9 \mathrm{H}), 1.00(\mathrm{~s}, 9 \mathrm{H}), 1.09(\mathrm{~s}, 3 \mathrm{H}), 1.22(\mathrm{~s}, 3 \mathrm{H}), 1.11-1.25(\mathrm{~m}, 2 \mathrm{H}), 1.28(\mathrm{dd}, J=3.5,10.2 \mathrm{~Hz}, 1 \mathrm{H}), 1.36$ (m, $2 \mathrm{H}), 1.54-1.70(\mathrm{~m}, 3 \mathrm{H}), 1.89(\mathrm{~m}, 1 \mathrm{H}), 1.97(\mathrm{dd}, J=2.7,12.4 \mathrm{~Hz}, 1 \mathrm{H}), 2.56(\mathrm{~m}, 1 \mathrm{H}), 3.11(\mathrm{~d}, J=$ $9.7 \mathrm{~Hz}, 1 \mathrm{H}), 3.20(\mathrm{dd}, J=7.4,10.4 \mathrm{~Hz}, 1 \mathrm{H}), 3.33$ (s, $3 \mathrm{H}), 3.43(\mathrm{dd}, J=5.1,10.5 \mathrm{~Hz}, 1 \mathrm{H}), 3.45(\mathrm{~d}, J=$ $9.7 \mathrm{~Hz}, 1 \mathrm{H}), 3.80(\mathrm{dd}, J=4.7,10.9 \mathrm{~Hz}, 1 \mathrm{H}) ;{ }^{13} \mathrm{C} \mathrm{NMR}\left(125 \mathrm{MHz}, \mathrm{C}_{6} \mathrm{D}_{6}\right) \delta 176.1,78.0,66.6,64.6$, $53.8,50.7,44.1,40.6,39.9,36.8,32.4,27.9,26.2,26.1,26.0,21.3,18.4,18.2,18.0,13.2,11.9,-3.5,-$ 4.7, -5.2, -5.6. HRMS (ESI) $\mathrm{m} / \mathrm{z} 565.3742\left[(\mathrm{M}+\mathrm{Na})^{+}\right.$; calcd for $\mathrm{C}_{29} \mathrm{H}_{58} \mathrm{O}_{5} \mathrm{NaSi}_{2}$ : 565.3720].

Preparation of the Eastern Hemisphere Subtarget (3aR,5aR,6R,7S,9aS,9bS)-7-(tertbutyldimethylsilyloxy)-6-((tert-butyldimethylsilyloxy)methyl)-6,9a,9b-trimethyldecahydro naphtho[1,2-c]furan-1(9bH)-one [(+)-6].

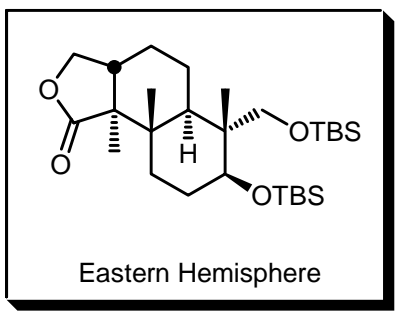

A $100 \mathrm{~mL}$ round bottom flask was charged with hydroxyester $(+)-30(1.08 \mathrm{~g}$, $2.0 \mathrm{mmol}, 1$ equiv) and THF (30 mL). The resulting solution was cooled to 0 ${ }^{\circ} \mathrm{C}$ with an ice-water bath and the $1.0 \mathrm{M}$ LiHMDS solution in THF $(2.4 \mathrm{~mL}$, 2.4 mmol, 1.2 equiv) was added slowly while maintaining an internal temperature no higher than $4{ }^{\circ} \mathrm{C}$. The resulting yellow solution was allowed to warm to room temperature in 35 minutes. The reaction mixture was then quenched with saturated aqueous $\mathrm{NH}_{4} \mathrm{Cl}$ solution $(40 \mathrm{~mL})$. The THF was removed from the resulting biphasic mixture in vacuo and brine (50 $\mathrm{mL})$ was added. The aqueous phase was extracted with $\mathrm{Et}_{2} \mathrm{O}(1 \mathrm{x} 100 \mathrm{~mL}$, then 2 × $50 \mathrm{~mL})$. The combined organic layers were dried over $\mathrm{MgSO}_{4}$ and concentrated in vacuo to give a white solid 
residue. The crude product was subjected to column chromatography (Hexanes : $\mathrm{Et}_{2} \mathrm{O}=3: 2$ ) and the product was obtained as a white waxy solid: $968 \mathrm{mg}(95 \%) . \mathrm{mp}=149-150{ }^{\circ} \mathrm{C} .[\alpha]_{\mathrm{D}}^{20}+1.6^{\circ}(c 0.63$, $\left.\mathrm{CHCl}_{3}\right)$.

IR (film) 2953.9 (s, br), 1760.7 (s), 1470.9 (m), 1386.3 (m), 1360.1 (m), 1248.7 (m), 1077.5 (m), 930.7 (m) $\mathrm{cm}^{-1} ;{ }^{1} \mathrm{H}$ NMR $\left(500 \mathrm{MHz}, \mathrm{CDCl}_{3}\right) \delta 0.01(\mathrm{~s}, 3 \mathrm{H}), 0.02(\mathrm{~s}, 6 \mathrm{H}), 0.04(\mathrm{~s}, 3 \mathrm{H}), 0.59(\mathrm{~s}, 3 \mathrm{H}), 0.85$ (s, 9 H), 0.89 (s, 9 H), 1.09 (s, 3 H), 1.14 (s, 3 H), 1.29 (m, 2 H), 1.50 (m, 2 H), 1.64 (m, 3 H), 1.91 (dd, J = 3.2, $12.3 \mathrm{~Hz}, 1 \mathrm{H}), 2.02$ (ddd, $J=3.4,3.4,13.4 \mathrm{~Hz}, 1 \mathrm{H}), 2.60(\mathrm{~m}, 1 \mathrm{H}), 3.05(\mathrm{~d}, J=9.8 \mathrm{~Hz}, 1 \mathrm{H}), 3.41$ (d, $J=9.8 \mathrm{~Hz}, 1 \mathrm{H}), 3.65(\mathrm{dd}, J=5.4,11.1 \mathrm{~Hz}, 1 \mathrm{H}), 3.88(\mathrm{dd}, J=8.4,11.6 \mathrm{~Hz}, 1 \mathrm{H}), 4.14(\mathrm{dd}, J=7.2$, $8.2 \mathrm{~Hz}, 1 \mathrm{H}) ;{ }^{13} \mathrm{C} \mathrm{NMR}\left(125 \mathrm{MHz}, \mathrm{CDCl}_{3}\right) \delta 179.9,71.7,69.2,64.3,49.8,43.7,40.1,38.0,37.0,29.5$, 27.3, 25.9, 25.9, 21.6, 21.5, 18.1, 17.9, 17.8, 12.2, 11.1, -3.5, -4.9, -5.3, -5.8. HRMS (ESI) $\mathrm{m} / \mathrm{z}$ found 533.3478 [(M+Na) ${ }^{+}$; calcd. for $\mathrm{C}_{28} \mathrm{H}_{54} \mathrm{O}_{4} \mathrm{NaSi}_{2}:$ 533.3458].

\section{REFERENCES}

(1) Burchat, A. F.; Chong, J. M.; Nielsen, N. J. Organomet. Chem. 1997, 542, 281-283.

(2) Ciceri, P.; Demnitz, F. W. J. Tetrahedron Lett. 1997, 38, 389-390.

(3) Bauduin, G.; Pietrasanta, Y. Tetrahedron 1973, 29, 4225-4231.

(4) Smith, A. B., III; Mewshaw, R. J. Org. Chem. 1984, 49, 3685-3689. 